\title{
Pollenkörner am Bienenkörper nach der Bestäubung verschiedener Kohlsorten (Brassica oleracea)
}

\author{
S Kubišová *, H Háslbachová \\ College of Agriculture, Brno, Tschechoslowakei
}

(eingegangen 15 Dezember 1988; angenommen 7 August 1989)

\begin{abstract}
Zusammenfassung - Auf Kohlblüten sammelnde Bienen hatten nach einer Nacht im Volk nur wenige, meist abgestorbene Poilenkörner an ihrem Körper (Überlebensrate 0,5-1,5\%). Die bevorzugten Körperstellen sind auf den schematischen Zeichnungen im Text dargestellt. Die Lebensfähigkeit des Pollens nimmt innerhalb von 1 zu 2 Stunden stark ab, auf den Bienen schneller als in den Staubbeuteln $(P=0,01)$. Auf Bienen, die im Käfig gehalten wurden, fanden sich mehr Pollenkörner mit höherer Überlebensrate als auf Bienen aus dem Volk. Auf Grund dieser Ergebnisse kann angenommen werden, daß dieselben Bienen schon an aufeinanderfolgenden Tagen zur Bestäubung unterschied-lichen Zuchtmaterials verwendet werden können.
\end{abstract}

\section{Apis mellifera / Pollen / Lebensfähigkeit / Bestäubung / Brassica oleracea}

\section{EINLEITUNG}

Beim Anbau eines Hybridsaatgutes von autoinkompatiblen Linien oder Weibchenlinien mit cytoplasmatischer Pollensterilität ist es dringend nötig, eine Vielzahl von Bienen-Bestäubungseinheiten für die Pollenübertragung einzusetzen. Es war erforderlich, diese Bienenvölkchen mehrmals zur Bestäubung eines genetisch verwandten Materials auszunutzen. Die Ansichten über die Zeitdauer, die zur Ausschaltung der Möglichkeit unerwüschter Befruchtung durch den auf den Bienenkörpern haftenden Pollen erforderlich ist, sind unterschiedlich. In den während vier Sommern wiederholten biologischen Versuchen haben wir ermittelt, da $B$ die zur Befruchtung eines genetisch verwandten Materials von Brassica oleracea benutzten Bienen selbst nach einer nur zwölfstündigen Isolation (d.h. nach einer Nachtpause) keine Befruchtung verursacht haben (Háslbachová et al, 1986). Lespinasse und Chevreu (1984) stellten jedoch im Gegensatz dazu bei Kreuzungen von Apfelklonen fest, daß die Bienen offensichtlich nach der Nachtpause doch den unerwüschten Pollen übertragen haben.

* Korrespondenz und Sonderdrucke. 
In der vorliegenden Arbeit waren wir bemüht zu ermitteln, ob die Bienen vor dem ersten Ausflug aus den Bienenstöcken nach der Nachtpause noch Pollen am Körper tragen, wo und in welchen Mengen, und ob es wahrscheinlich ist, daß dieser Pollen die von den Bienen besuchten Blütennarben in ausreichender Menge erreichen kann. Wir verfolgten auch die Abnahme der Lebensfähigkeit des Pollens auf dem Körper der Bienen, die vom Bienenstock für die Dauer von 0-22 Stunden nach dem Abfangen im Pflanzenbestand isoliert wurden, oder auch auf dem Körper der Bienen, die im Bienenstock übernachtet haben. In der wissenschaftlichen Literatur fanden wir nur Angaben über die Lebensfähigkeit des Pollens auf dem Bienenkörper, unmittelbar nach dem Fangen im Bestand (Parker und Hatley, 1979) oder bis zu 2-3 Stunden nach dem Abfangen (Buchareva, 1971).

\section{MATERIAL UND METHODIK}

Die diesbezüglichen Versuche wurden während der Jahre 1983-1987 im Forschungs- und Züchtungsinstitut für Gemüsebau in Olomouc, Morava durchgeführt. Als Versuchsmaterial dienten einerseits autoinkompatible Kohllinien, andererseits pollenfertile Blumenkohl- (Brassica oleracea var botrytis) und Kohlrabilinien ( $B$. oleracea var gongylodes). Die Versuchspflanzen wurden in Tunnelisolationsanlagen aus Silonnetz $(20 \times 3,8 \mathrm{~m})$ oder in Isolationsgewächshäusern $(5 \times 2,5 \mathrm{~m})$ gepflanzt. In jeder Isolationsanlage wurde während der Blütezeit ein Bienenvölkchen von $250 \mathrm{~g}$ oder $1 \mathrm{~kg}$ (nach Größe des Isolationsraumes) ohne Bienenkönigin, jedoch mit Brut (1 bis zu $10 \mathrm{dm}^{2}$ resp) untergebracht. Das Bienenalter schwankte von einer bis zu fünf Wochen.

\section{Untersuchung des Pollenvorkommens auf dem Bienenkörper}

Einen Tag vor der mikroskopischen Untersuchung wurden die in den Isolationsräumen befruchtenden Bienen gefangen, am Thoraxschild mit einer Farbe gezeichnet und wieder freigelassen; die Kontrollbienen wurden gleich nach dem Fangen mikroskopiert. Am nächsten Tag zwischen 6 und 7 Uhr wurden die gezeichneten Bienen wieder direkt von den Waben abgefangen (10 bis 60 Stück), mittels Äthyläther getötet und unter dem Stereomikroskop bei 25-85 maliger Vergrößerung untersucht. Das Vorkommen von Pollen wurde nach Lokalisation und Ablagerungsweise auf den Bienenkörpern graphisch dargestellt und an den mittels Rastermikroskop REM Tesla BS 300 aufgenommenen Photographien erfaßt.

\section{Lebensfähigkeit des Pollens auf dem Bienenkörper}

Während der Jahre 1983 und 1984 wurden die Versuchsbienen direkt an den Blüten des Kohls zwischen 10 und $11 \mathrm{Uhr}$ abgefangen und lebend einzeln in Eprouvetten gebracht. Täglich wurden 15 Bienen abgefangen, von 5 Bienen wurde der Pollen nach einer Stunde, von weiteren 5 nach 2-3 Stunden und von den übrigen nach 5-6 entnommen. Eine andere Gruppe von 5 Bienen wurde auf den Blüten um 17 Uhr abgefangen und in einem kleinen Käfig mit Honig und Wasser bis zum Morgen des nächsten Tages, d.h. 15-16 Stunden lang, aufbewahrt. Im Jahre 1984 wurden vormittags zusätzlich 5 Bienen abgefangen und in kleinen Käfigen dann bis zum nächsten Tag, $d$ h 21-22 Stunden lang, belassen. In beiden Jahren wurde das Abfangen der Bienen je dreimal wiederholt, im Jahre 1983 in zwei Serien, im Jahre 1984 in einer Serie. Gleichzeitig mit dem Abfangen der Bienen wurde Pollen direkt von reifen Staubbeuteln mehrerer Pflanzen entnommen und in Petrischalen als eine Mischprobe aufbewahrt. Aus diesen Schalen wurde im Jahre 1983 der Pollen sogleich zum Mikroskopieren entnommen, im Jahre 1984 in denselben Zeitintervallen wie von den Bienen.

Im Jahre 1985 und 1987 wurden die Versuchsbienen bei der Arbeit auf den Blüten nur gezeichnet und wieder freigelassen. Am zweiten Tag um 7 Uhr früh wurden diese Bienen direkt aus den Bienenstöcken entnommen und zur Abnahme des Pollens getötet. 


\section{Vorbereitung der Pollenpräparate und Ermittlung der Lebensfähigkeit des Pollens}

Der von verschiedenen Stellen des Körpers der Versuchsbienen entnommene Pollen wurde auf einem Objektträger in einen Tropfen Fluoreszenzdiazetat gebracht und unter dem Mikroskop mit Fluoreszenzeinrichtung bei einer hundertfachen Vergrößerung untersucht. Die Lebensfähigkeit des Pollens wurde durch den Nachweis aktiver Esterasen mittels fluorchromatischer Reaktion nach Heslop-Harrison und Heslop-Harrison (1970) ermittelt. Von der Gesamtanzahl untersuchter Pollerikörner wurde dann der Prozentsatz der leuchtenden, d.h. der lebensfähigen Pollenkörner berechnet. Während der Jahre 1985 und 1987 wurden in den vorbereiteten Pollenpräparaten zuerst sämtliche Pollenkörner im optischen Mikroskop gezählt und dann die leuchtenden Pollenkörner mit der Fluoreszenzeinrichtung bestimmt.

\section{ERGEBNISSE}

\section{Lokalisation des Pollens auf dem Bienenkörper}

Die Lokalisation des Pollens auf dem Bienenkörper verfolgten wir immer auf Bienen, die während der Tage mit höchster Arbeitsaktivität gefangen wurden. Wir untersuchten im Juni und Juli 1984 insgesamt 179 Versuchsbienen (nach der Nachtpause) und 30 Kontrollbienen (gleich nach dem Abfangen auf den Blüten).

Die Pollenkörner kamen an bestimmten charakteristischen Körperteilen der Bienen vor, und zwar entweder einzeln oder in Anhäufungen. Die Pollenkörner erschienen weißlich und oval, so wie die von den Staubbeuteln entnommenen Pollenkörner, oder gelblich und kugelförmig, also wesentlich verändert im Vergleich zu den aus Staubbeuteln entnommenen Körnern. Im ersten Fall handelt es sich wahrscheinlich um Pollenkörner, die von den Bienen während der Arbeit auf den Blüten am selben oder vorigen Tag aufgefangen wurden, im zweiten Fall befanden sich wahrscheinlich diese Pollenkörner schon seit längerer Zeit auf den Bienenkörpern. Die charakteristischen Pollenfundstellen sind aus den Abbildungen 1-12 ersichtlich.

Auf der Kopfvorderseite (Abb 1) wurden Pollenkörner einzeln in den Härchen zwischen den Fühlern an der Stirn der Versuchs- sowie Kontrollbienen gefunden. Diese waren klein und gelblich; bei der Entnahme wurden auch die Härchen abgerissen. Unter den Härchen am Vertex hatten die Kontrollbienen weissliche Pollenkörner, die Versuchsbienen wiesen fast keine Pollenkörner auf.

Auf der Kopfhinterseite (Abb 2 und 3) fanden wir bei den Kontrollbienen

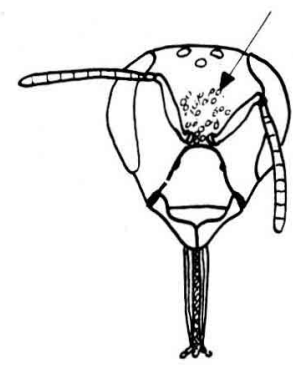

Abb 1. Bienenkopf von vorne. Die Lage der Pollenkörner ist mit Pfeilen bezeichnet.

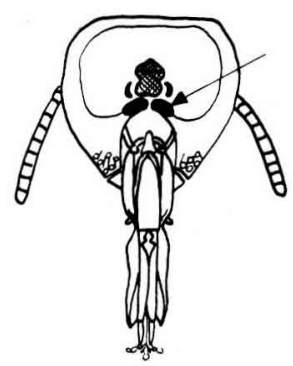

Abb 2. Bienenkopt von hinten. Legende wie in Abb 1. 
weißliche Pollenkörner auf den Postgenalhärchen. Die meisten Bienen beider Gruppen hatten die Pollenkörner in gelblichen Anhäufungen in Vertiefungen unter dem Foramen occipitale verklebt.

Bei den meisten Bienen beider Gruppen wurde eine beträchtliche Menge des angehäuften Pollens an der Ventralseite des Thorax in einer Vertiefung der Rille der Mesosternums ermittelt. Ganz kleine Pollenkörner kamen um die Coxae des zweiten und dritten Beinpaares vor. Bei fast allen Bienen waren die Pollenkörner in den Härchen direkt am Thoraxskellett zerstreut, namentlich am Lateralteil von Metathorax und Propodeum (Abb 4-5).

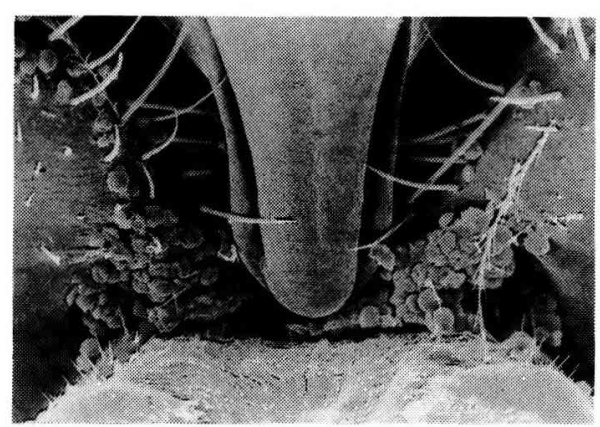

Abb 3. Anhäufung von Pollenkörnern unterhalb des Foramen occipitale (x 210).

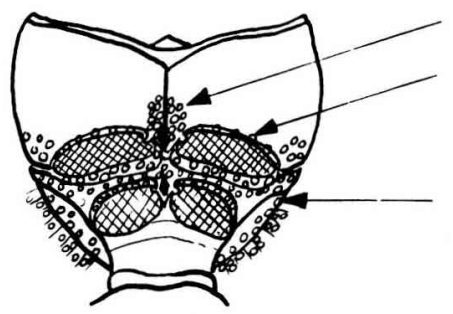

Abb 4. Ventralseite von Meso- und Metathorax und Propodaeum. Legende wie in Abb1.
Unter den Härchen der ersten drei Glieder aller Beinpaare befanden sich fast immer Pollenkörner, bei den Versuchsbienen in einer kleineren Menge als bei den Kontrollbienen. Auffallend waren gelbe Anhaüfungen der verklebten Pollenkörner auf dem Schenkel des zweiten Beinpaares (Abb 8). Regelmäßig wiesen die Bienen angehäufte Pollenkörner zwischen den Härchenreihen der Bürste am Tarsus des dritten Beinpaares auf (Abb 9). Bei den meisten Bienen kam ein wenig Pollen an der Unterseite des proximalen Flügelteiles vor, namentlich um die Costa herum (Abb 10). Am Abdomen kamen einzelne weißliche Pollenkörner in den Härchen von Sterniten der Kontrollbienen

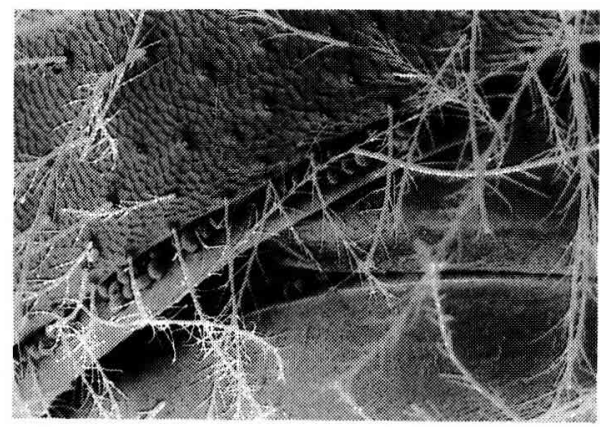

Abb 5. Pollenkörner in einer Spalte beim Hüftgelenk des 2. Beines (x 210).

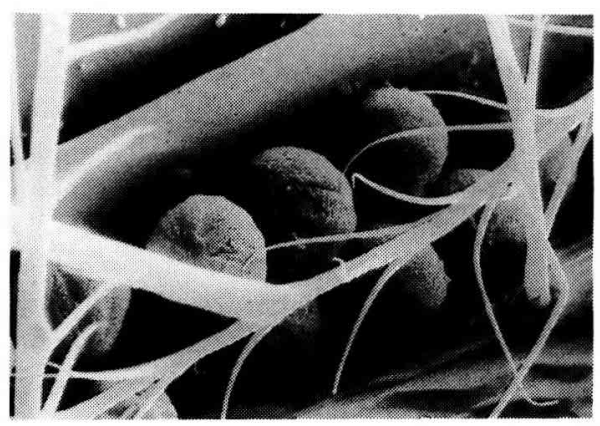

Abb 6. Detail aus Abb 5 ( $\times 1500)$. 

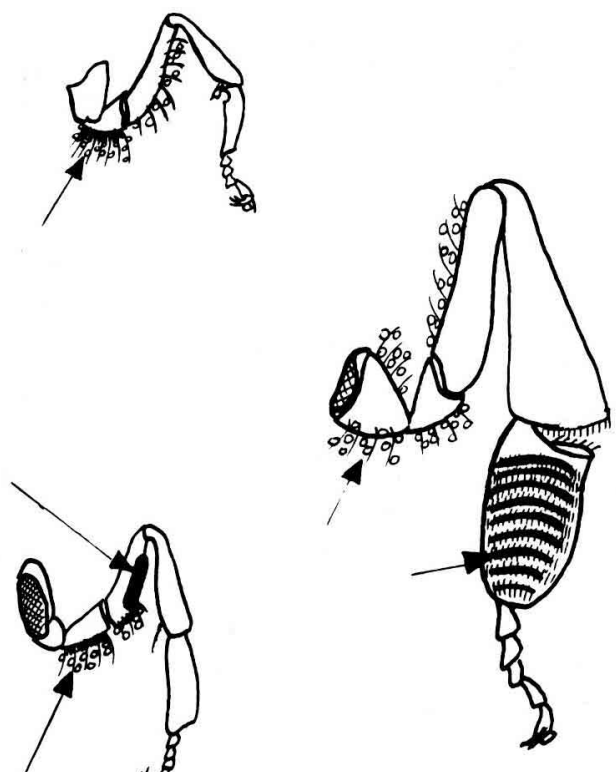

Abb 7. 1.-3. Bein. Legende wie in Abb1.

vor, bei den Versuchsbienen gelbliche Pollenkörner in einer dünnhäutigen Rille unterhalb der Wachsspiegel (Abb 12).

Wir haben festgestellt, daß nach einer Nachtpause auf den Bienen nach dem Beflug von Kohl oder Blumenkohl bedeutend weniger Pollen verblieben war,

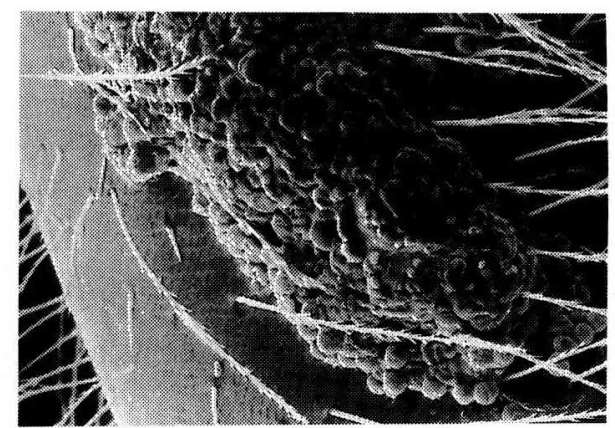

Abb 8. Anhäufung verklebter Pollenkörner an der Schenkelinnenseite (2. Bein) (x 210). als auf Kontrollbienen, die wir gleich nach dem Abfangen im Bestand mikroskopiert haben; es ist sehr unwahrscheinlich, daß diese Pollenkörner am nächsten Tag von diesen Bienen wieder auf die Blüten geraten könnten, aber diese Möglichkeit kann dennoch nicht ausgeschlossen werden.

\section{Lebensfähigkeit des Pollens auf dem Bienenkörper}

Im Jahre 1983 wurden insgesamt 110 Bienen in zwei Versuchsserien untersucht; 109 Bienen wurde der Pollen entnommen, und zwar in Intervallen zwischen 0 und 16 Stunden nach dem Abfangen und Isolieren außerhalb des Bienenvolkes. In der ersten Serie wurden durchschnittlich 36 Pollenkörner, in der zweiten 90 Pollenkörner je Biene untersucht.

Im Jahre 1984 wurden in einer Serie 75 Bienen untersucht, dabei wurden von 71 Bienen durchschnittlich 83 Pollenkörner je Biene gewonnen. Aus Pollen von Staubbeuteln wurden insgesamt 68 Mischproben hergestellt. Aus Abb 13 ist eine allmähliche Abnahme der lebensfähigen Pollenkörner ersichtlich. In Tabelle I und II sind Testergebnisse dargestellt, die die statistische Signifikanz dieser Abnahme überprüfen.

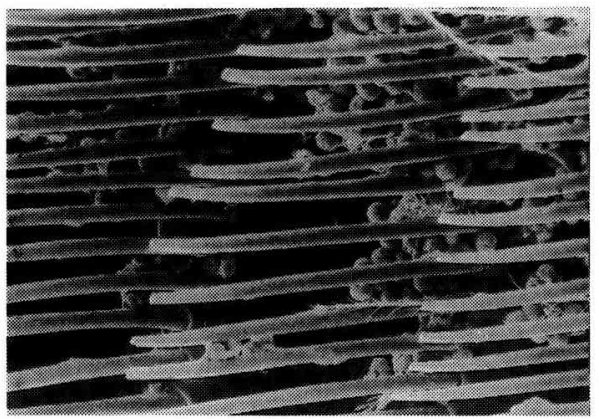

Abb 9. Pollenkörner zwischen den Borsten des Pollenkammes (3.Bein) (x 210). 
Tabelle I. Abnahme der Lebenskraft der Pollenkörner im Jahre 1983. * Die mit einer Vollinie unterstrichene Werte sind am Signifikanzniveau $P=0,05$ undifferenzierbar.

\begin{tabular}{|c|c|c|c|c|c|}
\hline \multirow[t]{3}{*}{ Versuchs serie } & \multirow{3}{*}{$\begin{array}{c}\text { von Staubeuteln } \\
0-1 \mathrm{~h}\end{array}$} & \multicolumn{3}{|c|}{ \% lebensfähiger Pollenkörner in Pollenprober } & \multirow[b]{3}{*}{$15-16 h$} \\
\hline & & \multicolumn{3}{|c|}{ von Bienen } & \\
\hline & & $0-1 h$ & $2-3 h$ & $5-6 h$ & \\
\hline 1 & 83,1 & 67,5 & $56,3^{*}$ & $53,6^{*}$ & 11,4 \\
\hline 2 & 73,7 & 34,0 & $28,8^{*}$ & $29,9^{*}$ & 19,8 \\
\hline
\end{tabular}

Tabelle II. Abnahme der Lebenskraft der Pollenkörner im Jahre 1984. Legende wie bei I. Tabelle.

\begin{tabular}{|c|c|c|c|c|c|c|}
\hline \multirow[t]{2}{*}{ Versuchsserie } & \multirow[t]{2}{*}{ Pollenherkunft } & \multicolumn{5}{|c|}{ \% lebensfähiger Pollenkörner in Pollenproben } \\
\hline & & $0-1 h$ & $2-3 h$ & $5-6 h$ & $21-22 h$ & $15-16 h$ \\
\hline \multirow[t]{2}{*}{3} & von Staubbeuteln & 59,3 & 54,1 & 46,4 & 41,4 & 40,2 \\
\hline & von Bienen & 36.3 & 33,3 & 18,9 & 16.7 & 8,6 \\
\hline
\end{tabular}

In allen drei Versuchsserien war die Lebensfähigkeit des Pollens auf den Bienenkörpern im Vergleich zur Lebensfähigkeit des Pollens aus den Staubbeuteln in gleichen Zeitintervallen immer statistisch signifikant niedriger $(P=0,01)$.

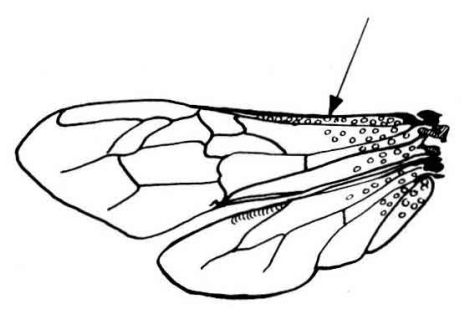

Abb 10. Bienenflügel mit verstreuten Pollenkörnern. Legende wie in Abb. 1.
In den Versuchen der Jahre 1985 und 1987 hatten die gezeichneten Bienen, die am Morgen aus den Bienenvölkern entnommen wurden, auf den Körpern weniger Pollen, als Bienen, die in den Jahren vorher im Pflanzenbestand abgefangen und einzeln gehalten wurden; nur bei wenigen gezeichneten Bienen war es möglich, überhaupt Pollen abzunehmen. Diese Tatsache ist aus Tabelle III ersichtlich. Untersucht wurden 40 Bienen, jedoch nur 17 von ihnen hatten Pollen in einer abnehmbaren Menge, und zwar vor allem am Thorax und an den Bürsten des dritten Beinpaares. Der Prozentsatz lebensfähiger Pollenkörner war bei diesen Versuchen sehr niedrig $(0,5-1,5 \%)$. 
Tablelle III. Lebenskraft der Pollenkörner auf den Bienen aus Bienenstöcken (1985 und 1987).

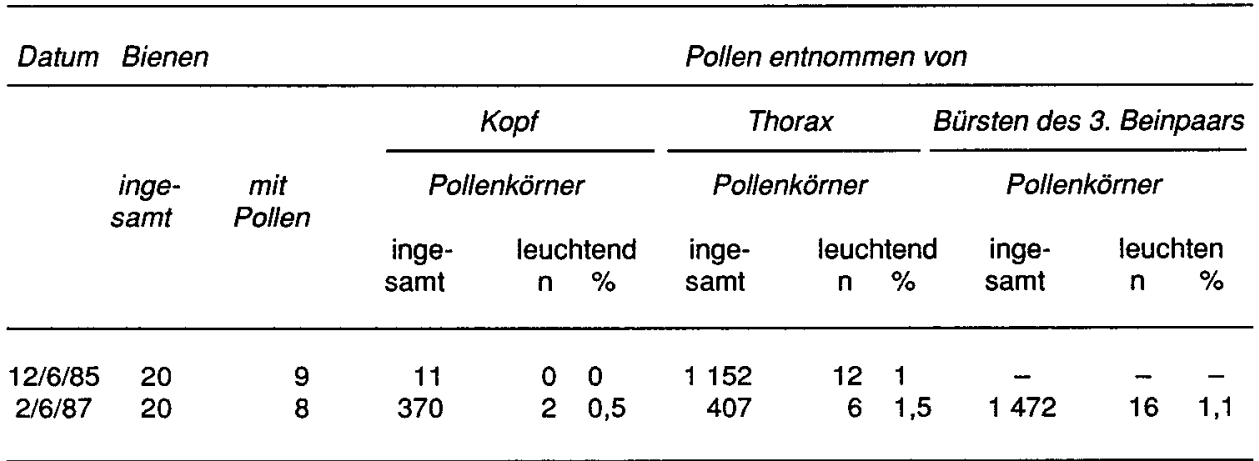

\section{DISKUSSION UND SCHLUBFOLGERUNG}

Die am Morgen aus den Bienenvölkern entnommenen Bienen hatten Pollenkörner hauptsächlich an schwer erreichbaren Körperstellen, von denen sie diese offensichtlich nicht selbst auskämmen konnten. Da diese Bienen nur sehr wenige Pollenkörner auf dem Körper hatten, mußten wir diese von allen Körperteilen abnehmen. Diese Pollenkörner befanden sich wahrscheinlich schon eine ziemlich

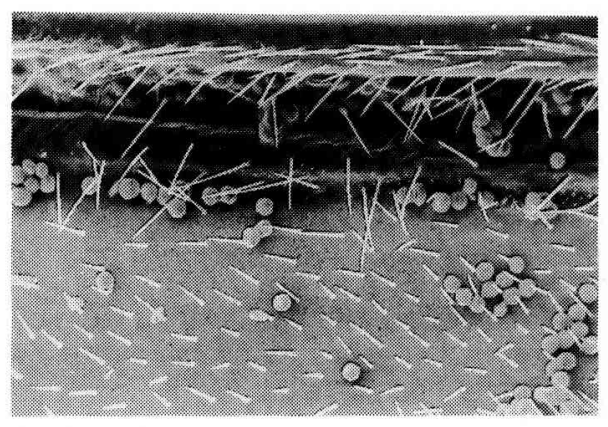

Abb 11. Teil des costalen und medialen Flügelfeldes von unten $(\times 210)$. lange Zeit auf den Bienenkörpern und waren deshalb meistens abgestorben.

Auf den während einer bestimmten Dauer außerhalb des Bienenstockes belassenen Bienen wurden mehr Pollenkörner gefunden: von den am schwersten zugänglichen Stellen des Bienenkörpers war der Pollen meistens nicht entfernt worden. Seine Lebensfähigkeit war höher, denn er war offensichtlich erst während der letzten Stunden vor dem Abfangen gesammelt worden.

Wir nehmen an, daß es im Milieu des Bienenvolkes zum schnelleren Verlust der Lebensfähigkeit der Pollenkörner kommt als auf Bienen, die außerhalb des Bienenstockes isoliert waren (vergleiche

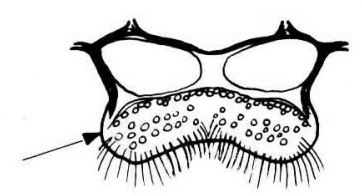

Abb 12. 5.Sternit des Abdomens. 


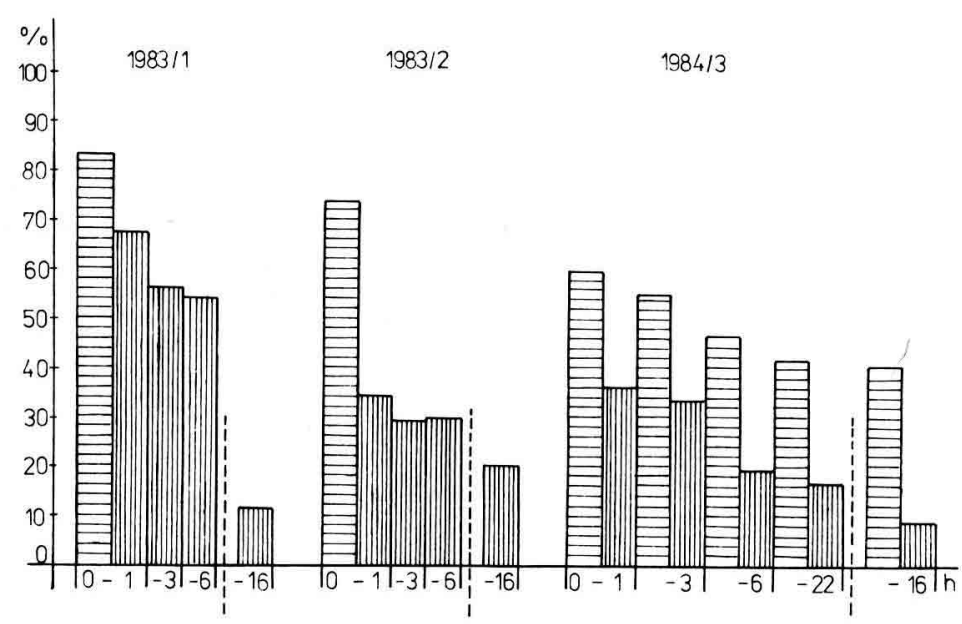

Abb 13. Lebensfähigkeit von Pollenkörnern aus den Staubbeuteln (waagrecht schraffierte Säulen) und vom Körper der Bienen (vertikal schraffierte Säulen). X-Achse: Zeitintervall der Bestimmung der Lebensfähigkeit des Pollens, Y-Achse: \% lebender Pollenkörner.

Tabelle I und II mit Tabelle III). Angesichts der kleinen Anzahl von Pollenkörnern, die auf den Bienen nach einer Nacht im Bienenstock verblieben sind und angesichts der geringen Lebensfähigkeit dieser Pollenkörner ist es sehr unwahrscheinlich, daß einige der Körner noch am nächsten Tag auf der Narbe haften und auskeimen können. Bei Gemüse der Art Brassica oleracea ist es deshalb möglich, dieselbe Bienengruppe für die Befruchtung eines unterschiedlichen Zuchtmaterials an aufeinanderfolgenden Tagen, bei nur einer Nachtpause, zu benutzen. Diese Tatsache haben auch unsere biologischen Tests gezeigt (Haslbachova et al, 1986).

Summary - Pollen grains adhering to bodies of honeybees after cole (Brassica oleracea) crop pollination. From 1983 to 1987 , the occurrence of pollen grains on honeybee bodies during and after pollination of cole crops and the viability of these pollen grains were observed. The objective of the study was to determine the shortest time lapse after which the same group of honeybees could be utilized for pollination of genetically related material without the risk of causing undesirable crossfertilization.

The honeybees were marked while at work on flowers. The morning of the following day, they were caught on combs before leaving the hive (tested bees). Using a stereomicroscope, the occurrence of pollen grains on their bodies was ascertained and the loci of occurrence and the mode of pollen deposition were recorded; details were represented in drawings and photographs made with the use of a REM Tesla BS 300 microscope. The honeybees used as controls were examined in the same way immediately after they were caught on flowers. Pollen grains on bodies of honeybees were much less numerous on tested bees than on control ones. The figures show the localization of pollen grains: head (figs 1-3), ventral face of thorax (fig 4-6), 
pairs of legs (figs 7-9, wing (fig 10 and 11), 5th sternit (fig 12).

The viability of pollen grains found on the bodies of honeybees was tested on individuals caught on flowers; groups of five were isolated for $1,3,5,15$ and 22 hours. There after, the pollen was wiped from their bodies and its viability assessed by the proof of active esterases due to fluorochromatic reaction, following the method described by Heslop-Harrison and HeslopHarrison (1970). Pollen removed from anthers and stored in Petri dishes was also tested at identical time intervals. From 1985 to 1987 , honeybees caught on flowers were color-marked and taken from the hive the following day for examination. Viability of pollen grains decreased with time away from anthers, the decline being more rapid $(P=0.01)$ in pollen on bodies of honeybees (fig 13: columns with vertical bars) than it was in pollen from anthers (fig 13: columns with horizontal bars). The viability of pollen grains found on the bodies of honeybees isolated for 15-22 hours outside the hive was always higher (9-20\%) than it was on bees taken from the hive $(0.5-1.5 \%)$, the former carrying appreciably larger amounts of pollen grains than the latter.

It is concluded that, in the studied plant material, transportation of viable pollen carried on bodies of honeybees from the hive to the stigmas of flowers is highly improbable, because of the low germinability of the pollen grains and their deposition on inaccessible parts of the honeybee body.

Apis mellifera / pollen / viability / pollination / Brassica oleracea

Résumé - Les grains de pollen présents sur le corps des abeilles pollinisatrices des choux (Brassica oleracea). De 1983 à 1987 on a observé la présence de grains de pollen sur le corps des abeilles pendant et après la pollinisation des choux et étudié leur viabilité. Le but de l'étude était de déterminer la période de temps la plus courte au bout de laquelle le même groupe d'abeilles pouvait être utilisé pour polliniser du matériel génétiquement apparenté sans risque d'hybridation indésirable.

Les abeilles ont été marquées lorsqu'elles butinaient les fleurs. Le matin du jour suivant, elles ont été prélevées sur les rayons avant qu'elles ne quittent la ruche (abeilles testées). La présence de grains de pollen sur leur corps a été vérifiée à l'aide d'un stéréomicroscope; les endroits et le mode de dépôt du pollen ont été notés à l'aide de dessins et de photographies faites au microscope électronique à balayage Tesla BS 300 . Les abeilles servant de témoins ont été examinées de la même façon juste après avoir été capturées sur les fleurs. Les grains de pollen présents sur le corps des abeilles sont bien moins nombreux chez les abeilles testées que chez les témoins. Les figures montrent la localisation des grains de pollen: tête (figs 1-3), face ventrale du thorax (fig 4-6), paire de pattes (figs 7-9), aile (figs 10 et 11 ), $5^{\mathrm{e}}$ sternite (fig 12).

On a étudié la viabilité des grains de pollen présents sur le corps des abeilles en train de butiner. Les abeilles ont été capturées sur les fleurs puis isolées par groupes de 5 durant $1,3,5,15$ et 22 heures. Le pollen a été ensuite ôté du corps des abeilles et sa viabilité déterminée par la présence d'estérases actives à l'aide de la réaction fluorochromatique selon la méthode de Heslop-Harrison et Heslop-Harrison (1970). A titre de contrôle, du pollen prélevé sur les stigmates et mis en boîtes de Petri a été testé à des intervalles de temps identiques. En 1985 et 1987, les abeilles capturées sur les fleurs ont été marquées puis relachées et capturées dans la ruche le lendemain matin. La 
viabilité des grains de pollen a diminué plus rapidement avec le temps $(P=0,01)$ pour le pollen présent sur le corps des abeilles (fig 13, colonnes rayées verticalement) que pour celui des anthères (fig 13, colonnes rayées horizontalement). La viabilité a toujours été plus élevée (9-20\%) pour les grains de pollen prélevés sur le corps des abeilles isolées pendant 15 et 22 heures hors de la ruche, que pour celui prélevé sur les abeilles de la ruche $(0,5-$ $1,5 \%)$.

Conclusion : pour le matériel végétal étudié, il est fortement improbable que des grains de pollen viables puissent être transportés sur le corps des abeilles depuis la ruche jusque sur les stigmates après une nuit passée dans la ruche, en raison de leur faible viabilité et de leur présence à des endroits peu accessibles du corps des abeilles.

Apis mellifica / pollen / viabilité / pollinisation / Brassica oleracea

\section{LITERATUR}

Buchareva GA (1971) Pollen and bees (in Russian) Pchelovodstvo (6) 17-18

Háslbachová $H$, Kubišová $S$, Matẹjík T, Śmrhová O (1986) Study on the transfer of pollen by bees after isolation (in Czech) $S b$ UVTIZ, Zahradnictvi 13, 299-306

Heslop-Harrison J, Heslop-Harrison Y (1970) Evaluation of pollen viability by enzymatically induced fluorescence, intracellular hydrolysis of fluoresceindiacetate. Stain Technol 45, 115-120

Lespinasse $Y$, Chevreu E (1984) Utilisation d'un gène marqueur pour la recherche de plantes haploïdes de pommier : comparaison entre pollinisation manuelle et pollinisation par les abeilles. CR Symp Int Pollinisation, Versailles 1983, INRA, Paris, 391-396

Parker FD, Hatley CL (1979) Onion pollination: viability of onion pollen and pollen diversity on insect body hairs. Proc IVth Int Symp on Pollination, Maryland, 1978, 201-206 